\title{
Differentiation of Erysipelothrix rhusiopathiae and Erysipelothrix tonsillarum by Sodium Dodecyl Sulfate- Polyacrylamide Gel Electrophoresis of Cell Proteins
}

\author{
YUTAKA TAMURA, ${ }^{1 *}$ TOSHIO TAKAHASHI, ${ }^{1}$ KAMALUDDIN ZARKASIE, ${ }^{2}$ \\ MASAYUKI NAKAMURA, ${ }^{1}$ AND HARUO YOSHIMURA ${ }^{1}$ \\ National Veterinary Assay Laboratory, 1-15-1 Tokura, Kokubunji, Tokyo 185, Japan ${ }^{1}$ and \\ Veterinary Drug Assay Laboratory, Gunungsindur, Bogor 16340, Indonesia ${ }^{2}$
}

\begin{abstract}
The protein patterns of whole cells of Erysipelothrix rhusiopathiae and Erysipelothrix tonsillarum were studied by using sodium dodecyl sulfate-polyacrylamide gel electrophoresis. The protein patterns of the 16 strains of $E$. rhusiopathiae and $E$. tonsillarum studied, including the type strains of these two species, resembled each other, except that there were 71-, 41-, 34-, and 26-kDa proteins in the $E$. rhusiopathiae pattern and 74-, 44-, 36-, and 25-kDa proteins in the $E$. tonsillarum pattern. This observation indicates that there is some phenotypic heterogeneity in the genus Erysipelothrix. In addition, the protein patterns of $E$. rhusiopathiae serotype reference strains representing serotypes 1 through 23 and type $N$ were compared. The protein patterns of serotype $1 \mathrm{a}, 1 \mathrm{~b}, 2,4,5,6,8,9,11,12,15,16,19$, and 21 and type $N$ strains were similar to the pattern of the type strain of $E$. rhusiopathiae (strain ATCC 19414). Conversely, the protein patterns of serotype 3, 7, 10, 14, and 20 strains were very similar to the pattern of the type strain of $E$. tonsillarum (strain ATCC 43339). An atypical pattern was observed in serotype 13, 17, 18, 22, and 23 strains. These results suggest that this method may be used as an aid in studying the taxonomy of these bacteria.
\end{abstract}

Erysipelothrix rhusiopathiae is the causative agent of swine erysipelas (15). This organism is also found in the tonsils of healthy swine, in slime on fish, and in the environment. E. rhusiopathiae strains are classified into serotypes 1 (with subtypes $1 \mathrm{a}$ and $1 \mathrm{~b}$ ) through 23 and type $\mathrm{N}$; type $\mathrm{N}$ strains do not produce any precipitating antibody against homologous and heterologous heat-stable extracts in rabbits $(6,11)$. The genus Erysipelothrix was originally thought to consist of a single species despite the wide range of hosts and wide variety of serological characteristics of $E$. rhusiopathiae (2).

Recently, we reported that a cluster of avirulent serotype 7 strains found in porcine tonsils was genetically distinct from $E$ rhusiopathiae, and a new species was proposed for serotype 7 strains, Erysipelothrix tonsillarum (8). Moreover, serotypes 1 through 23 and type $\mathrm{N}$ of $E$. rhusiopathiae were studied, and two distinct DNA relatedness groups were identified on the basis of the results of DNA-DNA hybridization experiments performed with the type strains of these two species (9). However, phenotypically, E. tonsillarum appeared to be identical to $E$. rhusiopathiae except that it was able to produce acid from saccharose $(8,9)$.

Since cell proteins are genetically directed, their electrophoretic patterns tend to express genetic relationships among microorganisms (7). The purposes of this study were to compare the electrophoretic patterns of whole-cell proteins from strains of E. rhusiopathiae and E. tonsillarum and to identify phenotypic characteristics that distinguish these two species.

\section{MATERIALS AND METHODS}

Bacterial strains. Eight field strains identified phenotypically and genetically as $E$. rhusiopathiae (representing serotypes $2,6,11,12$, and 16) and six field strains identified

\footnotetext{
* Corresponding author.
}

phenotypically and genetically as $E$. tonsillarum (representing serotype 7$)(8,10)$ were used. These strains were isolated from tonsils of apparently healthy slaughter swine in 1984. The type strains of $E$. rhusiopathiae (strain ATCC 19414) (serotype 2) and E. tonsillarum (strain ATCC 43339) (serotype 7) were obtained from the American Type Culture Collection, Rockville, Md. To compare the protein patterns of all of the serotypes, serotype reference strains identified phenotypically as $E$. rhusiopathiae representing serotypes 1 through 23 and type $N(9,11)$ were also used. These strains segregated into two main DNA-DNA hybridization groups, E. rhusiopathiae and E. tonsillarum (9). Serotyping of the strains was performed by the method described previously $(6,11)$.

SDS-PAGE. To obtain cells for sodium dodecyl sulfatepolyacrylamide gel electrophoresis (SDS-PAGE), colonies from a 48-h agar plate culture of each strain were inoculated into tryptose phosphate broth (Difco) ( $\mathrm{pH} \mathrm{7.6).} \mathrm{After} \mathrm{incu-}$ bation for $48 \mathrm{~h}$ at $37^{\circ} \mathrm{C}$, the broth culture was centrifuged at $12,000 \times g$ for $20 \mathrm{~min}$. The bacterial cells were washed three times with physiological saline and were suspended in 62.5 $\mathrm{mM}$ Tris hydrochloride buffer ( $\mathrm{pH}$ 6.8) containing 5\% 2-mercaptoethanol, $2 \%$ sodium dodecyl sulfate, $10 \%$ glycerol, and $0.002 \%$ bromphenol blue. SDS-PAGE was performed in $10 \%$ slab gels by the method of Laemmli (5). Approximate molecular masses were determined by comparing migration patterns with the migration patterns of proteins having known molecular masses (Fluka AG, Buchs, Switzerland). The gels were stained with Coomassie brilliant blue R-250.

Antisera. Antisera were prepared by hyperimmunizing rabbits with whole cells of the type strains of $E$. rhusiopathiae and $E$. tonsillarum (11).

To prepare strain-specific antisera, anti-ATCC $19414^{\mathrm{T}}$ (T $=$ type strain) serum and anti-ATCC $43339^{\mathrm{T}}$ serum were each absorbed with the heterologous type strain by using the procedure to remove cross-reactive antibodies described below. Formalinized cells of strains ATCC $19414^{\mathrm{T}}$ and ATCC $43339^{\mathrm{T}}$ were washed three times with physiological 


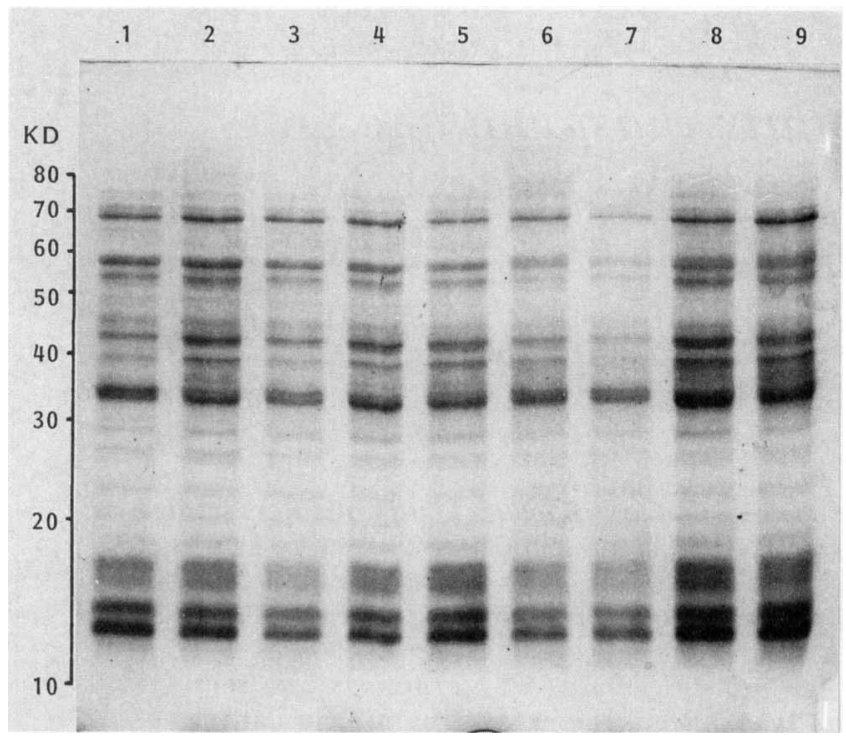

FIG. 1. SDS-PAGE patterns of porcine strains of $E$. rhusiopathiae. Lane 1, strain ATCC $19414^{\mathrm{T}}$ (serotype 2); lane 2, strain T-183 (serotype 2); lane 3, strain T-191 (serotype 2); lane 4, strain T-194 (serotype 2); lane 5, strain T-221 (serotype 2); lane 6, strain T-324 (serotype 6); lane 7, strain T-205 (serotype 11); lane 8, strain T-312 (serotype 12), lane 9, strain T-184 (serotype 16). KD, kilodaltons.

saline and were mixed with antisera at a concentration of 100 $\mathrm{mg} / \mathrm{ml}$. After incubation at $37^{\circ} \mathrm{C}$ for $2 \mathrm{~h}$ and then at $4^{\circ} \mathrm{C}$ for 18 $\mathrm{h}$, the antisera were separated by centrifugation. This procedure was repeated at least three times until the $O$ antibodies against heterologous antigen disappeared. The resulting sera were stored at $-20^{\circ} \mathrm{C}$ until they were used.

Western blotting (immunoblotting). Bacterial proteins were transferred to nitrocellulose membranes by electrophoresis at $100 \mathrm{~V}$ for $1 \mathrm{~h}$ with a transblot apparatus (Bio-Rad Laboratories, Richmond, Calif.), using a modification of the Western blot method of Towbin et al. (12). After the strips were treated with specific anti-ATCC $19414^{\mathrm{T}}$ serum or anti-ATCC $43339^{\mathrm{T}}$ serum, they were washed with Tris-buffered saline $(20 \mathrm{mM}$ Tris, $500 \mathrm{mM}$ sodium chloride; $\mathrm{pH}$ 7.2), incubated for $1 \mathrm{~h}$ with horseradish peroxidase-conjugated anti-rabbit immunoglobulin G goat serum (Cappel), and washed again. The strips were developed by using a color development reagent containing 4-chloro-1-naphthol (Bio-Rad).

\section{RESULTS}

Electrophoretic patterns of $\boldsymbol{E}$. rhusiopathiae strains. The protein patterns obtained for whole cells of $E$. rhusiopathiae strains isolated from tonsils and type strain ATCC 19414 are shown in Fig. 1. Our interpretation of the specific patterns was based on molecular weights $\left(R_{f}\right.$ values) rather than on the intensities of the bands. The protein patterns of the $E$. rhusiopathiae strains contained approximately 20 bands, 10 of which were consistently more prominent in Coomassie brillant blue-stained gels. The major proteins had molecular masses of $68,56,54,41,38,32,16,15,13$, and $12 \mathrm{kDa}$. Within E. rhusiopathiae, there was a good deal of similarity among the major proteins of nine strains, including the type strain.

Electrophoretic patterns of $\boldsymbol{E}$. tonsillarum strains. Figure 2 shows the protein patterns of six E. tonsillarum strains and type strain ATCC 43339; these strains were isolated from

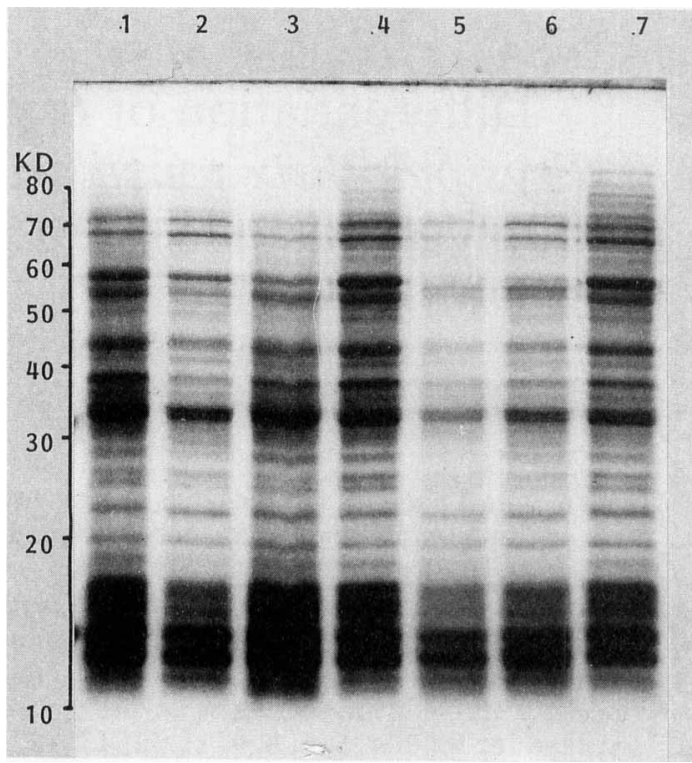

FIG. 2. SDS-PAGE patterns of porcine strains of $E$. tonsillarum. Lane 1, strain ATCC $43339^{\mathrm{T}}$; lane 2, strain T-228; lane 3, strain T-278; lane 4, strain T-285; lane 5, strain T-334; lane 6, strain T-339, lane 7, strain T-342. All of these strains belong to serotype 7. KD, kilodaltons.

tonsils. The protein patterns of E. tonsillarum strains contained approximately 24 bands, 11 of which were consistently more prominent in Coomassie brillant blue-stained gels. The major proteins had molecular masses of $74,68,56$, $54,44,38,32,16,15,13$, and $12 \mathrm{kDa}$. The patterns were similar for all strains of $E$. tonsillarum, including the type strain.

Comparison of the protein patterns of $E$. rhusiopathiae and $E$. tonsillarum. The protein patterns of E. rhusiopathiae and E. tonsillarum were compared (Fig. 3). The main differences in the protein patterns occurred in the 71-, 41-, 34-, and 26-kDa proteins of $E$. rhusiopathiae and the 74-, 44-, 36-, and $25-\mathrm{kDa}$ proteins of $E$. tonsillarum. To determine whether proteins detected by SDS-PAGE are specific for each species, specific proteins were examined by Western blot analysis. Figure 4 shows the reactions of specific antisera against the type strains of the two species with the preparations described above. Anti-ATCC $19414^{\mathrm{T}}$ serum reacted strongly with the 56- and 32-kDa protein bands from E. rhusiopathiae ATCC $19414^{\mathrm{T}}$ and T-194 preparations. No reaction between this serum and the $56-\mathrm{kDa}$ protein of $E$. tonsillarum was observed. In contrast, anti-ATCC $43339^{\mathrm{T}}$ serum reacted broadly with the $32-\mathrm{kDa}$ region from E. tonsillarum ATCC $43339^{T}$ and $T-278$ preparations. No reaction between this serum and the 32-kDa protein of E. rhusiopathiae was observed.

Comparison of the protein patterns of $E$. rhusiopathiae strains representing all serotypes. The protein patterns of $E$. rhusiopathiae serotype reference strains representing all 23 serotypes were compared with the protein patterns of the $E$. rhusiopathiae and E. tonsillarum type strains. The comparison of the protein patterns was carried out by examining the similarity in the patterns in the 73-, 43-, 35-, and $25-\mathrm{kDa}$ regions. The taxonomic relationship between serotypes and protein patterns in the genus Erysipelothrix is summarized in Table 1 . Strains representing serotypes 1 through 23 and type $\mathrm{N}$ segregated into two main groups on the basis of SDS-PAGE 


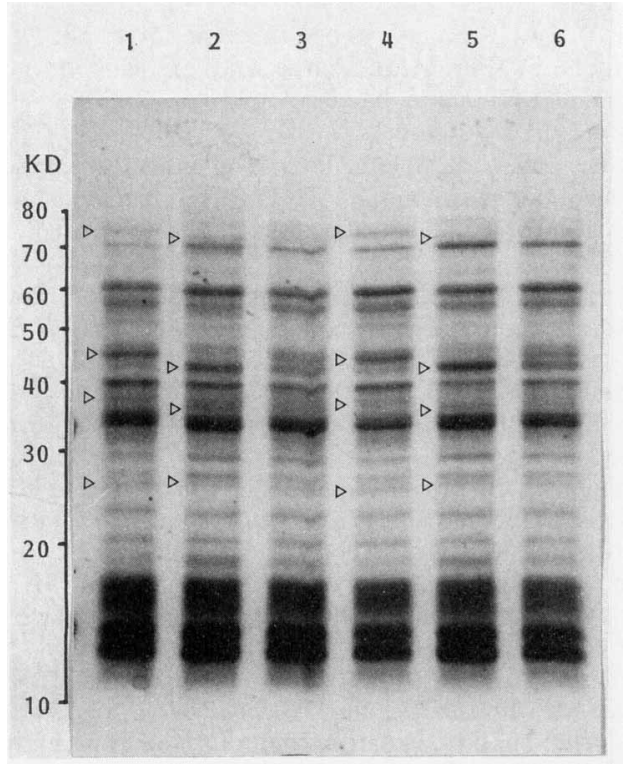

FIG. 3. Comparison of SDS-PAGE patterns of E. rhusiopathiae (lanes 2 and 5) and E. tonsillarum (lanes 1 and 4). Lane 1, strain ATCC $43339^{\mathrm{T}}$; lane 2 , strain ATCC $19414^{\mathrm{T}}$; lane 3 , strain ATCC $43339^{\mathrm{T}}$ plus strain ATCC $19414^{\mathrm{T}}$; lane 4 , strain T-285, lane 5 , strain T-194; lane 6, strain T-285 plus strain T-194. The arrowheads indicate the positions of different proteins of the two species. $\mathrm{KD}$, kilodaltons.

type. Strains belonging to serotypes $1 \mathrm{a}, 1 \mathrm{~b}, 2,4,5,6,8,9,11$, $12,15,16,19$, and 21 and type $\mathbf{N}$ produced patterns similar to the pattern of the type strain of $E$. rhusiopathiae. In contrast, strains belonging to serotypes $3,7,10,14$, and 20 produced patterns similar to the pattern of the type strain of $E$. tonsillarum. Strains belonging to serotypes $13,17,18,22$, and 23 produced independent protein patterns which seemed to be unrelated to the pattern of either type strain.

\section{DISCUSSION}

The results of this study of the protein patterns of whole cells of $E$. rhusiopathiae and $E$. tonsillarum shows that
TABLE 1. Classification in the genus Erysipelothrix

\begin{tabular}{llc}
\hline \multirow{2}{*}{ Species } & \multicolumn{2}{c}{ Serotypes and type as determined by: } \\
\cline { 2 - 3 } & \multicolumn{1}{c}{ Genetic techniques $^{a}$} & SDS-PAGE \\
\hline E. rhusiopathiae & $1 \mathrm{a}, 1 \mathrm{~b}, 2,4,5,6,8$, & $1 \mathrm{a}, 1 \mathrm{~b}, 2,4,5,6$, \\
& $9,11,12,15,16$, & $8,9,11,12,15$, \\
& $17,19,21, \mathrm{~N}$ & $16,19,21, \mathrm{~N}$ \\
E. tonsillarum & $3,7,10,14,20,22$, & $3,7,10,14,20$ \\
$\begin{array}{l}\text { Other Erysipelothrix } \\
\text { species }\end{array}$ & 13,18 & $13,17,18,22,23$ \\
\end{tabular}

${ }^{a}$ Strains belonging to serotypes $1 \mathrm{a}, 1 \mathrm{~b}, 2,4,5,6,8,9,11,12,15,16,17,19$, and 21 and type $N$ exhibited 73 to $103 \%$ DNA relatedness to the type strain of $E$. rhusiopathiae and 18 to $24 \%$ DNA relatedness to the type strain of $E$. tonsillarum. Strains belonging to serotypes $3,7,10,14,20,22$, and 23 exhibited 66 to $100 \%$ DNA relatedness to the type strain of $E$. tonsillarum and 14 to $27 \%$ DNA relatedness to the type strain of $E$. rhusiopathiae. Strains belonging to serotypes 13 and 18 exhibited low levels of hybridization (16 to $47 \%$ ) to both of the type strains (9).

under standard conditions, the SDS-PAGE system can provide highly reproducible results that differentiate these two species. The protein patterns of E. rhusiopathiae and $E$. tonsillarum are species specific and correlate with serological type. This is surprising since serological specificity is due to soluble peptidoglycans of the cell walls (3), which are not involved in protein determinants in SDS-PAGE. E. rhusiopathiae strains produce a pattern that is distinct from the $E$. tonsillarum pattern in the 73-, 43-, 35-, and $25-\mathrm{kDa}$ regions. We attempted to identify the specific proteins of these two species by Western blot analysis. Anti-ATCC $19414^{\mathrm{T}}$ serum specifically reacted with 56-kDa proteins from the type strain of $E$. rhusiopathiae, while anti-ATCC $43339^{\mathrm{T}}$ serum reacted broadly with $32-\mathrm{kDa}$ proteins from the type strain of $E$. tonsillarum. These results indicate that $E$. tonsillarum can be distinguished from $E$. rhusiopathiae by both SDS-PAGE and Western blot analysis, as well as by the ability of $E$. tonsillarum to produce acid from saccharose.

Recently, we reported that two distinct DNA relatedness groups were found in the genus Erysipelothrix (9). Interestingly, the results of our comprehensive SDS-PAGE studies demonstrate that $E$. rhusiopathiae strains representing sero-

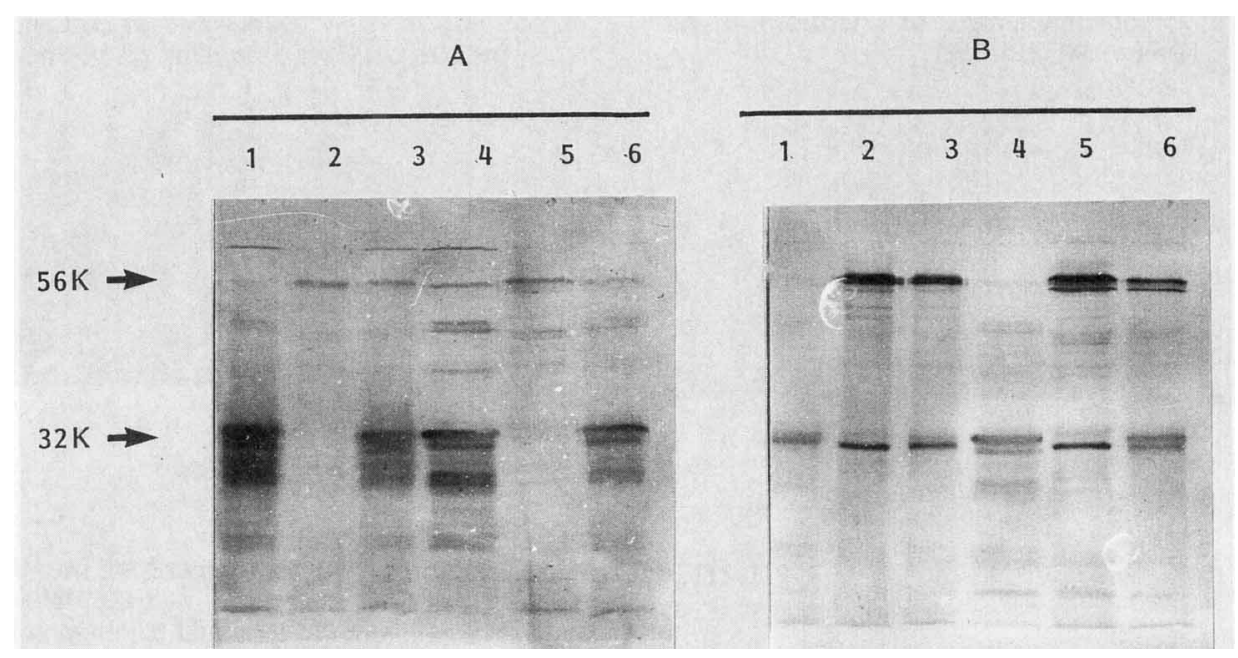

FIG. 4. Western blot analysis of anti-ATCC $19414^{\mathrm{T}}$ serum (B) and anti-ATCC $43339^{\mathrm{T}}$ serum (A) against the following antigens: strain ATCC $43339^{\mathrm{T}}$ (lanes 1); strain ATCC $19414^{\mathrm{T}}$ (lanes 2); strain ATCC $43339^{\mathrm{T}}$ plus strain ATCC 19414 ${ }^{\mathrm{T}}$ (lanes 3); strain T-278 (lanes 4); strain T-194 (lanes 5); strain T-278 plus strain T-194 (lanes 6). 
types 1 through 23 and type $\mathbf{N}$ derived from various sources also fall into two distinct groups. However, atypical patterns were obtained for strains belonging to serotypes $13,17,18$, 22 , and 23. Additional studies on the protein patterns of these serotypes will be necessary to elucidate the phenotypic differentiates among species in the genus Erysipelothrix.

White and Mirikitani reported that the protein patterns of $E$. rhusiopathiae strains were quite different, so that the SDS-PAGE method was judged to be unreliable for taxonomic purposes (13). Lachmann and Deicher reported that of the 20 separate bands obtained for $E$. rhusiopathiae T-28, two main proteins were found to migrate at molecular masses of 64 and $48 \mathrm{kDa}$ (4). Recently, an immunoblot analysis showed that antisera against the recombinant protein that protected mice against challenge with $E$. rhusiopathiae recognized proteins having molecular masses of 66 and $43 \mathrm{kDa}$ in whole-cell extracts and in Triton X-100solubilized surface proteins of $E$. rhusiopathiae (1). Taking the information described above into consideration, our results show that $E$. rhusiopathiae contains two major protein antigens having molecular masses of 32 and $56 \mathrm{kDa}$, which were readily detectable by anti-ATCC $19414^{\mathrm{T}}$ serum. In contrast, $E$. tonsillarum, which is not virulent, contains only a $32-\mathrm{kDa}$ protein that can be recognized with antiATCC $43339^{\mathrm{T}}$ serum. Therefore, the $56-\mathrm{kDa}$ protein may play an important role in the pathogenesis of $E$. rhusiopathiae. To elucidate these points, additional studies of the 56-kDa protein of $E$. rhusiopathiae will be needed.

Our previous attempts to determine the serotypes of $E$. rhusiopathiae strains obtained from swine with various clinical symptoms of erysipelas (11) and from apparently healthy swine (10) showed that most strains belong to serotypes 1 and 2 , as described by Wood (14). Strains belonging to these serotypes were experimentally highly virulent for swine and capable of inducing generalized urticarial lesions with depression and anorexia. On the other hand, most strains other than serotype 1 and 2 strains were nonvirulent or weakly virulent for swine. Therefore, the SDS-PAGE method in our study provided additional evidence that isolates belonging to serotypes 1 and 2 are indeed different from some strains belonging to nonvirulent serotypes.

\section{REFERENCES}

1. Galán, J. E., and J. F. Timoney. 1990. Cloning and expression in Escherichia coli of a protective antigen of Erysipelothrix rhusiopathiae. Infect. Immun. 58:3116-3121.
2. Jones, D. 1986. Genus Erysipelothrix, p. 1245-1249. In P. H. A. Sneath, N. S. Mair, M. E. Sharpe, and J. G. Holt (ed.), Bergey's manual of systematic bacteriology, vol. 2. The Williams \& Wilkins Co., Baltimore.

3. Kalf, G., and T. G. White. 1963. The antigenic components of Erysipelothrix rhusiopathiae. II. Purification and chemical characterization of a type-specific antigen. Arch. Biochem. Biophys. 102:39-47.

4. Lachmann, P. G., and H. Deicher. 1986. Solubilization and characterization of surface antigenic components of Erysipelothrix rhusiopathiae T28. Infect. Immun. 52:818-822.

5. Laemmli, U. K. 1970 . Cleavage of structural proteins during the assembly of the head of bacteriophage T4. Nature (London) 227:680-685.

6. Nørrung, V., B. Munch, and H. Errebo Larsen. 1987. Occurrence, isolation and serotyping of Erysipelothrix rhusiopathiae in cattle and pig slurry. Acta Vet. Scand. 28:9-14.

7. Razin, S., and S. Rottem. 1967. Identification of Mycoplasma and other microorganisms by polyacrylamide gel electrophoresis of cell proteins. J. Bacteriol. 94:1807-1810.

8. Takahashi, T., T. Fujisawa, Y. Benno, Y. Tamura, T. Sawada, S. Suzuki, M. Muramatsu, and T. Mitsuoka. 1987. Erysipelothrix tonsillarum sp. nov. isolated from tonsils of apparently healthy pigs. Int. J. Syst. Bacteriol. 37:166-168.

9. Takahashi, T., T. Fujisawa, Y. Tamura, S. Suzuki, M. Muramatsu, T. Sawada, Y. Benno, and T. Mitsuoka. 1992. DNA relatedness among Erysipelothrix rhusiopathiae strains representing all twenty-three serovars and Erysipelothrix tonsillarum. Int. J. Syst. Bacteriol. 42:469-473.

10. Takahashi, T., T. Sawada, M. Muramatsu, Y. Tamura, T. Fujisawa, Y. Benno, and T. Mitsuoka. 1987. Serotype, antimicrobial susceptibility, and pathogenicity of Erysipelothrix rhusiopathiae isolates from tonsils of apparently healthy slaughter pigs. J. Clin. Microbiol. 25:536-539.

11. Takahashi, T., T. Sawada, M. Takagi, K. Seto, M. Kanzaki, and T. Maruyama. 1984. Serotypes of Erysipelothrix rhusiopathiae strains isolated from slaughter pigs affected with chronic erysipelas. Jpn. J. Vet. Sci. 46:149-153.

12. Towbin, H. T., T. Staehelin, and J. Gordon. 1979. Electrophoretic transfer of proteins from polyacrylamide gels to nitrocellulose sheets: procedure and applications. Proc. Natl. Acad. Sci. USA 76:4350-4354.

13. White, T. G., and F. K. Mirikitani. 1975. Some biological and physical-chemical properties of Erysipelothrix rhusiopathiae. Cornell Vet. 66:152-163.

14. Wood, R. L. 1984. Swine erysipelas: a review of prevalence and research. J. Am. Vet. Med. Assoc. 184:944-948.

15. Wood, R. L., and R. D. Shuman. 1975. Swine erysipelas, p. 565-620. In H. W. Dunne and A. D. Leman (ed.), Diseases of swine, 4th ed. Iowa State University Press, Ames. 